(C)2008 IEEE. Personal use of this material is permitted. However, permission to reprint/republish this material for advertising or promotional purposes or for creating new collective works for resale or redistribution to servers or lists, or to reuse any copyrighted component of this work in other works must be obtained from the IEEE. 


\title{
Creating Interoperability Within Healthcare Industry
}

\author{
Chen Wu, Maja Hadzic \\ Research Lab for Digital Health Ecosystems, Curtin University of Technology, Perth, Australia \\ chen.wu@curtin.edu.au,m.hadzic@curtin.edu.au
}

\begin{abstract}
During the last decade, a number of health initiatives have been undertaken in Australia. However, Australian medical systems still suffer from the chronic problem of inability to share information essential to the health and wellbeing of patients. The major causes for this are (1) the lack of a standardized format in which patient information is being kept, and (2) the lack of infrastructure to enable sharing of the information among different organizations and institutions. In this paper we propose the use of ontologies, to enable effective translation between different EHR formats, and use of web services to enable efficient information exchange and sharing. The proposed solution has the potential to greatly improve the way patient information is being used, and consequently reduce the associated costs in both human and financial terms.
\end{abstract}

\section{INTRODUCTION}

Electronic Health Records (HER) systems are being implemented in a number of countries including America, Canada and UK [1]. HealthConnect [2] is the Australian government's EHR initiative which conducted a number of trials in 2002, 2003 and 2004 in Tasmania, Northern Territory, Queensland, New South Wales and South Australia. The project has shown success on a smaller scale but the major problem of sharing crucial patient information has not been solved. Every year more than 3,000 deaths in Australia are attributed to reasons that can be prevented with more effective e-health systems [3]. An ABC report [3] from November 2007 state that "one of the major killers of patients is the medical system's own chronic inability to share vital information".

Currently, a large number of medical practitioners in Australia use their own software to store and manage patient information. The format in which patient information is stored varies from one medical practitioner to another. Moreover, different medical institutions do not practice the exchange of patient information, even in situations of treating the same patient. We conclude that (1) the lack of a standardized format in which patient information is being kept, and (2) the lack of infrastructure to enable sharing of the information among different organizations and institutions, form two main obstacles in effective and efficient sharing of patient information.

Additional implementation barriers have been identified in [4]. These include financial expenditure, resistance to change, lack of office technology, lack of technological standards, increasing staff workloads and healthcare culture including attitude, workflows and relationships.

The effective EHR implementation and networking could save more than $\$ 81$ billion annually through improved health care efficiency and safety [5]. Moreover, the estimated savings could be doubled through the use of health information technology in prevention and management of chronic disease. Hillestad et al. [5] urge implementation of the required changes within the health care system. All hospitals, health institutions and organizations, and patients need to work together to identify mutually supportive roles that will enable them to move towards a shared vision.

A good solution to the current situation may be to not stay only focused on the outcomes such as (1) standardized electronic health records and (2) high capacity and quality infrastructure to support sharing of patient information, but also on the way we bring the current medical system into this new era of standardized and shared electronic health records. We do not intend to break the current practices; we respect the fact that many small scale medical communities flourish in different locations around the world. The least 'painful' change for medical practitioners would include keeping the complexity behind the screen. We believe that this can be achieved by giving the option to medical practitioners of keeping the same interface (which can be done by limiting the change to the back-end system), or changing the interface to match the standardized format. For medical practitioners resisting the change, the translation between different heath record formats will be done behind the screen and their view of the patient record will not change. This will give them a false appearance of the old system. In contrast, medical practitioners more receptive to change will choose the standard interface.

In this paper we will present an achievable solution for effective and efficient implementation of electronic health records within Australia. In Section 2, we will discuss the current two most popular EHR implementation efforts which use Smart Card and Radio-frequency identification (RFID) technologies. In Section 3, we will illustrate how ontologies can be used to mediate between different EHR formats. The system enabling sharing of patient information and its implementation will be described in Sections 4 and 5 respectively. The paper is concluded in Section 6.

\section{RELATED WORK}

The need for standardized or interpretable EHR is evident [6]. The two most popular standardization efforts use Smart Card and RFID technologies. These technologies support the creation of machine-readable medical records where the digital components are used to screen and read personal medical records.

A large number of companies provide Smart Health Card e.g. Almex Ltd. SmartCard Solutions, Smart Health 
Solutions, Electronia, and Giesecke \& Devrient. The Smart Cards differ from each other in the way they store, process, and manage information, and deal with the associated privacy and security issues. The choice has to be made carefully. Different factors must be considered, balancing between the Smart Card advantages and possible problems that may arise.

RFID for people has gained lots of attention in the last couple of years. Even though the Food and Drug Administration (FDA) approved VeriChip in 2003, they have reported a number of potential dangers associated with the use of VeriChip [7]. These include adverse tissue reaction, migration of the chip, failure of the chip, electrical hazards and magnetic resonance imaging (MRI) incompatibility. MRI incompatibility is particularly dangerous as it allows for the electrical currents to be induced in conductive metal implants resulting in severe patient burns.

In addition to health risks, the FDA has also identified a problem of compromised data security [7]. Criminals can use a reader device to capture information from an implanted chip as well as to duplicate this information and create a cloned chip with the same function. Additionally, the reader device can be used to track an individual. Katherine Albrech states [7] that "That tracking potential, coupled with VeriChip's potential health risks make the VeriChip a very poor choice for medical patients seeking safety and security".

Additional disadvantages of microchips have been reported in 2007 [8]. The formation of malignant sarcomas and other cancers around or adjacent to the implanted microchips have been reported. Overall it has been concluded that the microchips had induced the cancers.

The current Australian health system is marked by its heterogeneous nature. Having the frontier technologies in mind and global vision for the health care system, as well as the current needs of Australian patients and medical practitioners, we propose a solution that will lift up the Australian health system to the level where the interoperability of EHR and sharing of patient information will be possible. In the following two sections, we will discuss the technologies that can be implemented to realize this vision.

\section{Ontology Mediates BetweEn DiffEREnT EHR FORMATS}

One of the major barriers to adopting an EHR system [9] is the lack of a standard for interoperability among competing software options. Ontologies are a shared and formal conceptualization of specific domain knowledge and can be used for data standardization [10]. In this section we will explain how ontologies can be used for standardization of EHR. The preliminary idea is also illustrated in our previous work [11].

We illustrate the main idea on a simple example shown in Figure 1. The three different EHR (EHR1, EHR2 and EHR3) represent the different formats in which patient information is being kept. For example, EHR1 format is used by a public hospital, EHR2 by a private practice and EHR3 by a medical centre. Generally, all EHR capture very similar information, but the way this information is organized differs from one EHR to the other.

We introduce the use of ontologies as a way to mediate between the different EHR formats. The method of creating the mediating ontology consists of the following five steps:

(1) form an Ontology Committee consisting of representatives from each health institution using their own EHR format

(2) jointly identify synonyms, related information and overlaps between the different EHR formats

(3) choose the most appropriate terminology to represent the overlapping information and/or introduce new terminology where needed, and agree to use this terminology in a consistent and coherent manner (i.e. commit to this terminology) [9]

(4) formalize the agreed terminology creating Electronic Health Records Ontology (EHRO)

(5) map the concepts of individual EHR to the concepts of EHRO

As ontologies represent agreed knowledge shared within a specific community, it is required to form an Ontology Committee. This Ontology Committee will consist of two representatives from each EHR system community, one for the users and one for the software developers. In our example, the Ontology Committee would consist of at least six members with two representatives from each EHR system.

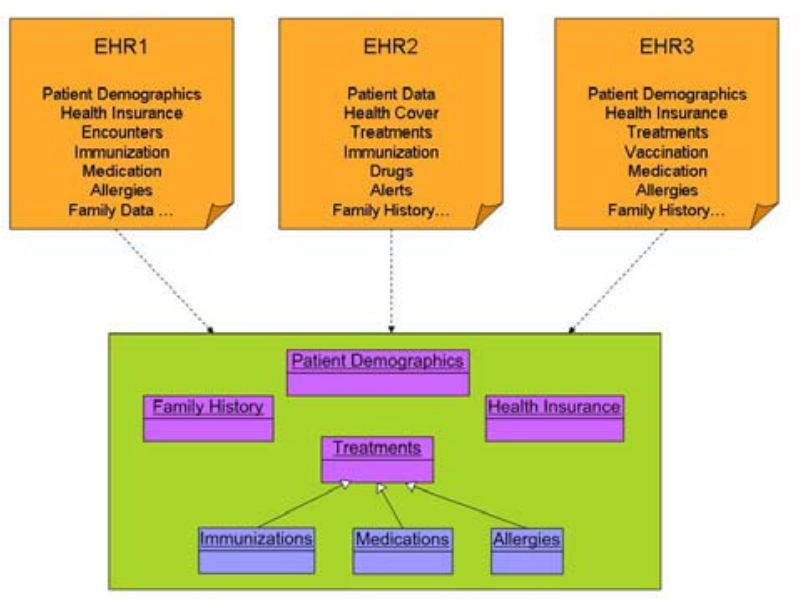

Fig. 1. Using the different EHR formats to create a shared EHRO

The Ontology Committee members jointly identify areas of overlaps between different EHR formats. This also includes identification of terms with the same meaning (synonyms). In our example, 'Patient Demographics' from EHR1 and 'Patient Data' from EHR2 collect and store identical information. The same holds true for 'Health Insurance' from EHR1 and 'Health Cover' from EHR2, 'Medications' from EHR1 and 'Drugs' from EHR2, etc. Different EHR systems may organize information differently, and the situation may not be as obvious as shown in Figure 1. In these situations it 
may be needed to group a number of related concepts under the umbrella of a more generic concept, and then identify possible overlaps. For example, patient 'Name', 'Date of Birth', 'Gender' and 'Address' can be grouped under the concept of 'Patient Demographics'.

Once the overlapping information has been identified, the Ontology Committee members need to choose appropriate names for the areas of overlaps. Practically, the most intuitive or the most frequently used term is chosen. In our example from Figure 1, the term 'Immunization' appears in EHR1 and EHR2 while 'Vaccination' appears in EHR3. We can agree to use the term 'Immunization' in EHRO as this term is used more frequently. Vaccination is the most common technique of immunization, but is not the only one. So, an additional advantage of using the term 'Immunization' is the broader meaning of this term. Coming to an agreement regarding the shared terminology can be a difficult and time-consuming task. Often, parties will compromise in order to establish a shared terminology framework which is more easily understood, as the achieving interoperable healthcare system has great value.

Once the terminology has been agreed upon by all Ontology Committee members, it needs to be formalized. Formalization of the selected terminology will enable the machines to access, read, understand and manipulate the formatted information. Different tools can be used to formalize the terminology i.e. to create EHRO. It is important to choose a toll that will enable creation of an ontology file compatible with the existing EHR formats, and allow mapping of EHR concepts to the EHRO concepts.

The last step is to establish the correspondence between EHR and the common EHRO. The concepts of the existing EHR need to be mapped to the EHRO concepts. This will allow translation between the different EHR formats. For example, if EHR1 is available to the EHR2 users, EHR1 concepts can be mapped to the EHRO and from EHRO into the EHR2 format. This kind of translation between different EHR formats can greatly contribute to the sharing of patient information between different institutions which use inconsistent EHR formats.

\section{ENABLING SHARING OF PATIENT INFORMATION}

In this section, we will discuss the architecture of a system that will support the sharing of patient information. This architecture can be implemented within a smaller region, and further extended to be implemented on a larger scale. In Figure 2, we illustrate the main principle behind the system and explain the mechanism in sequel of this section.

\section{A. EHR Syndication and EHR Peer}

An EHR Syndication is a focused community whereby a group of healthcare professionals, medical specialists and organisations who use the same EHR format, form a relatively stable community that continually provides services to their patients. The rationale of establishing EHR Syndication is based on the fact that there exist many different EHR formats (e.g. Almex Ltd. SmartCard Solutions, Smart Health Solutions, Electronia, and Giesecke \& Devrient are different companies which use different formats of Smart Health Cards). The different EHR formats have been adopted by different organisations resulting in formation of a number of syndication and an overall heterogeneous EHR structure. It would be very difficult (if it is not completely unfeasible) to mandate each one of them to adopt exactly the same EHR format. EHR Syndication provides an empirical way whereby health and medical organization who share common standard and interests follow the same rules and conventions in order to fulfil EHR requirements efficiently and add extra values to the healthcare community. Note that the notion of EHR Syndication is purely logical, and is thus orthogonal to the physical geographic locations. For example, two GPs from Australia and UK may end up joining the same EHR Syndication as long as they are willing to follow the same EHR format.

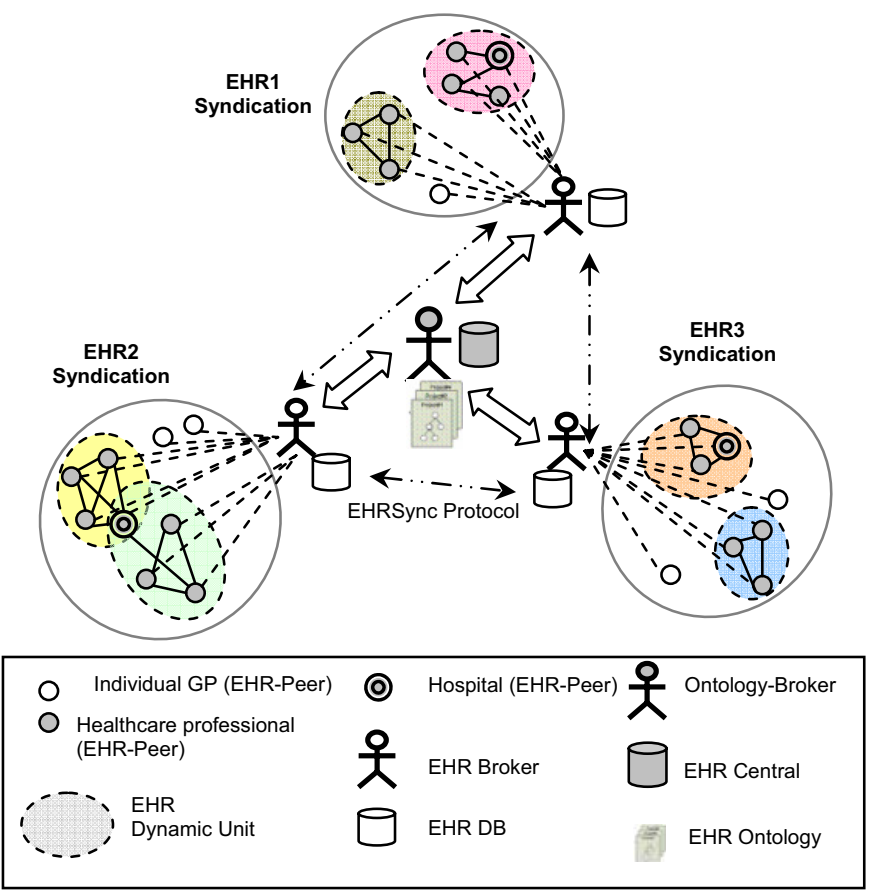

Fig. 2. Overall architecture of the interoperable healthcare system

An EHR Peer is a customisable software tool provided within the EHR Syndication that can interact with the EHR Broker in order to retrieve useful EHR data. Another very important use of EHR Peer is that once a health organization agrees to join the EHR Syndication, the EHR Peer is the data entry gateway, to which new EHR instance data is published from their legacy systems. Authorised health and medical professionals are also able to update the EHR DB through the EHR Peer user interface. In order to serve health and medical professionals working in various conditions (e.g. desktop, PDA, emergency, etc.), EHR Peer uses as much as possible open Web-based communication protocols (e.g. HTTP, 
Atom, SOAP, etc.) augmented with proper security mechanisms.

An EHR Dynamic Unit is a transient community that allows a small group of healthcare professionals, medical specialists and organisations from a particular EHR Syndication to form an ad-hoc team working collectively in order to diagnose and solve specific medical problems of patients.

\section{B. EHR Broker}

An EHR Broker is responsible for providing infrastructural services to health and medical organizations in an EHR Syndication, e.g. conducting EHR related activities within the EHR Syndication. EHR Broker plays a key role in servicing the local EHR Syndication members. Most EHR information (e.g. new patient records) can be periodically disseminated as the "syndication event" via the Publish/Subscribe mode. For example, when a medical professional attempts to search a particular disease symptom from all patients within the same syndication, it can subscribe to this special topic, which allows this medical practitioner to constantly stay updated through the EHR Peer whenever any new symptom is added to the EHR DB by other health and medical professionals. Figure 3 indicates our module design of EHR Broker, which consists of six core components: the EHR Broker Interface, the Publish/Subscribe Server, the Sync Server, the Matching Engine, the EHR DB, and the EHR Central API.

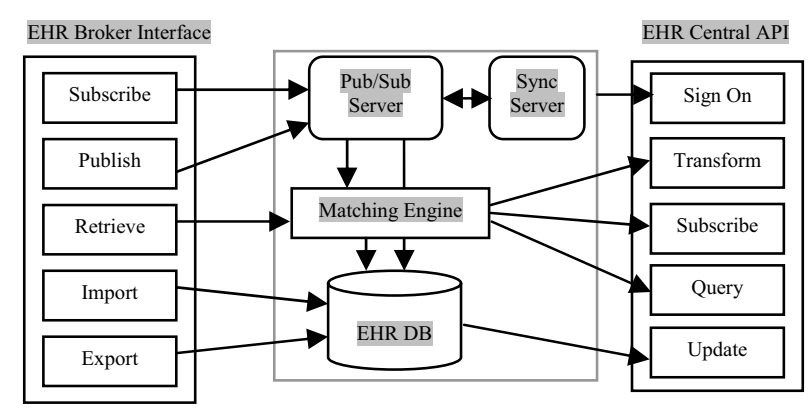

Fig. 3. EHR Broker architecture

The EHR Broker Interface accepts EHR requests from the EHR Peers and/or EHR Brokers, interprets and forwards the requests to different components of the EHR Broker. EHR Interface currently supports five types of EHR requests: Subscribe, Publish, Retrieve, Import, and Export.

The Subscribe request represents a subscription of EHR instance data. In general, two types of Subscribe requests are identified. The first one is sent from a normal EHR Peer, whose operator wants to find EHR data such as a patient's records, or a particular part of disease's detailed information. The second type of subscription is requested by another EHR Broker using the EHRSync protocol (see Figure 2), which in turn employs the subscription mechanism to synchronise the EHR data between two EHR Syndications. The Sync Server component will be resorted to process further issues relating to two-way resource synchronisation. The Sync Server also manages the subscription to medical records published through other EHR Brokers via the EHRSync Protocol. The EHRSync Protocol is a data communication protocol, by which two EHR syndications can synchronise EHR instance data in order to maintain the data consistency. The rationale behind the EHRSync Protocol is that it allows EHR data to be shared between different EHR syndications in a timely and reliable manner without going through the EHR Central. It is a direct two-way asynchronous peer-to-peer communication. Such a loosely-coupled, while efficiently inter-connected relationship amongst EHR Syndications increase the system scalability and reliability. For example, suppose EHR Peers from Figure 2 in EHR2 Syndication are very interested in a particular symptom caused by pneumonia, for research purposes. The EHR Broker in EHR2 Syndication thus subscribes to new patient records that have "pneumonia" from EHR1 Syndication. Whenever patients with pneumonia turn up in GPs from EHR1 Syndication, this information (i.e. the instance EHR data) will be automatically disseminated to EHR2 Syndication. Note that only instance data will be transferred, thus, if EHR2 Syndication does not understand the format of EHR1 Syndication's data, it has to resort to the EHR Central, which employs the EHR ontology to conduct data transformation.

The Publish request represents the EHR data changes that have occurred within the EHR Syndication. For example, a patient has visited a GP, who then updated this patient's medical record through the EHR Peer. This updated information will then be published to the EHR Broker as an event consequently updating the information available via EHR Central, enabling other interested EHR Syndications that have subscribed to such an event to access this information.

The Retrieve request represents the demand of medical professionals for finding particular useful/critical medical record information. The request is sent to the Matching Engine, which acts as a search engine that attempts to find the matched medical record or part of the medical record that meets the demand requirements. Note that retrieve request might be fired by EHR Brokers from other EHR Syndications. In this case, the patient's token is needed as an authorisation that permits the EHR Broker to release (a part of) a particular patient's medical records.

The Import and the Export requests are two utilities that allow the medical professionals to efficiently manipulate the medical records data in a batch mode. This is particularly useful when a new medical professional joins the EHR Syndication, to which a large number of new medical records will perhaps be imported from the EHR Peer operator.

The EHR Central API is essentially a set of software library that allows the EHR Broker to interact with the EHR Central. The Sign On API literally functions as a management mediator between the EHR Syndication and the EHR Central, dealing with bootstrapping and administrative issues such as syndication registry, metadata population, etc. To the EHR Broker, the Transform API can transform the medical records 
from one format/standard to another. However, it does not achieve this on its own, rather, it forwards the transformation request to the EHR Central which uses the EHR ontology to accomplish this important task. Through the Subscribe API, the EHR Broker is able to detect changes of metadata, which specifies the location of each patient's medical records that are appealing to this EHR Broker. The Query API works in the similar fashion as the Subscribe API except that it allows the EHR Broker to swiftly discover a particular patients' record regardless whether or not any changes have occurred in it. The Update API deals with changes that have occurred within the EHR Syndication. For example, a UK tourist attends an Australian GP due to illness during travelling. In this case, the local Australian EHR Syndication would need to update this incident to the EHR Central, informing that a part of this tourist's medical record lies in its own EHR DB.

An EHR DB is an EHR data storage in which the instance level data of EHR within the same EHR Syndication is kept and managed by the EHR Broker. It uses sophisticated indexing techniques to enhance the effectiveness and efficiency of the EHR retrieval conducted by each EHR Peer.

\section{EHR Central}

EHR Central maintains a central index that records the mapping between the patient's identity and EHR Syndications that contain his or her medical records. It acts as a hash table, where the key is the patient's identification (e.g. full name, date of birth, place of birth, etc.) and the value is the EHR Syndication's unique identifier. Note that one patient's full medical record might be split into several parts, each of which resides in a distinct EHR Syndication. For example, a UK student Jerry, who had medical records in London, needs to see a GP in Perth during his study in Curtin University. In this case, examining Jerry's full medical record requires access to two EHR Syndications if Jerry's doctors do not belong to the same EHR Syndication. Therefore, two records of Jerry's medical information will be kept in the EHR Central's central index (i.e. the hash table). Although the implementation of this hash table is beyond the scope of this paper, it is evident that sophisticated indexing techniques are highly desirable in order to support both patient-driven and syndication-driven queries. Following the above example, Jerry's doctor in Perth cannot access his medical records in London without Jerry's informed consent and authorisation. This way, the privacy problem can be partially avoided although the full treatment of privacy issues is beyond the scope of this paper, which focuses on the conceptual and architectural model of EHR solution.

EHR Central has two important architectural components: the EHR Ontology and the EHR Ontology Broker.

EHR Ontology (EHRO) is a shared conceptualisation of the EHR that enables translation between different EHR formats.

An Ontology broker is responsible for maintaining the EHRO and for transforming the EHR data representation from one format to another. It is also responsible for updating the EHRO, thereby realising the EHRO evolution based on both users' opinions and expert decisions. Any changes made to the EHRO will be forwarded to a related EHR Broker for the caching purpose.

\section{IMPLEMENTATION}

In this section, we discuss the implementation of the EHR architecture. The implementation will be built on top of open, proven, Web-based technologies. Two important principles that we have learnt from previous Web information systems will guide the implementation work - simplicity and ubiquity. By simplicity, we mean that EHR system users can easily use the system without having to install, configure, maintain, and learn complex software systems. This is consistent with the purpose to keep 'the least 'painful' change for the medical practitioners" as suggested in Section I. We also intend to leverage existing simple communication protocols, techniques, and algorithms as much as we can in order to reduce the complexity. By ubiquity, we mean that the underlying technology candidate has to be well accepted and supported amongst a broad range of users, devices, software libraries, legacy systems, IT infrastructures, etc.

TABLE I

THE MAPPING BETWEEN ATOM ENTRY AND EHR

\begin{tabular}{ll}
\hline \multicolumn{1}{c}{ Atom Entry } & \multicolumn{1}{c}{ EHR Metadata } \\
\hline atomAuthor & Patient's GP \\
\hline atomCategory & Illness type \\
\hline atomContent & Syndication-specific EHR markup \\
\hline atomContributor & $\begin{array}{l}\text { Additional doctors who contribute to } \\
\text { patient's treatment }\end{array}$ \\
\hline atomId & $\begin{array}{l}\text { Unique EHR ID generated by the } \\
\text { EHR system }\end{array}$ \\
\hline atomLink & $\begin{array}{l}\text { Link to the next and previous records } \\
\text { (if any) of the same patient }\end{array}$ \\
\hline atomPublished & $\begin{array}{l}\text { Time a record was published to EHR } \\
\text { Central }\end{array}$ \\
\hline atomRights & Privacy information \\
\hline atomSource & $\begin{array}{l}\text { Used for local cache purpose when a } \\
\text { record is copied from one syndication } \\
\text { to another }\end{array}$ \\
\hline atomSummary & $\begin{array}{l}\text { A short abstract of the patient's } \\
\text { treatment history }\end{array}$ \\
\hline atomTitle & Patient's name \\
\hline atomUpdated & Last time a record was updated \\
\hline
\end{tabular}

With these two principles, we have chosen HTTP 1.1 [12] as the fundamental communication protocol. As the key protocol underpinning the World Wide Web, the largest distributed system human kind has ever built, HTTP fully embraces the simplicity and ubiquity principles that are essential for building a scalable and simple EHR infrastructure. On top of HTTP, we use Atom [13] to represent the EHR instance data in order to realize the publish/subscribe model (see Figure 2 and 3). Atom standard 
defines an XML-based file format that allows lists of information, known as "feeds", to be syndicated between publishers and consumers. In addition to syndicating Weblogs, news, and other Web contents, Atom can be used for other important functions. Our previous work [14] has utilized Atom as the representation of Web services metadata to realize Web-aligned service discovery. Similarly, Table 1 provides a conceptual mapping between an Atom feed entry and the metadata of a medical record. Note that, in Table 1, each Atom entry is retreated as a segment of the full medical record of one patient (see the "UK student Jerry example" given in Section IV.C). Therefore, the atomLink element acts as a reference, pointing to both the previous and the next segments.

Web services technology is used to implement EHR Broker Interface and the EHR Central API. In particular, we use the RESTful Web services in conjunction with the Atom Publish Protocol (APP [15]) and the Java technology. Due to the page limit, in what follows, we will only illustrate the proposed implementation work for the EHR Broker as shown in Table 2. We use a number of open source software libraries to implement different components. At this stage, the prototype development is underway and we will conduct sets of experiments and field deployment in cooperation with local hospitals and health organizations in Western Australia.

TABLE II

THE EHR BROKER IMPLEMENTATION SOLUTION

\begin{tabular}{ll}
\hline \multicolumn{1}{c}{$\begin{array}{c}\text { Architectural } \\
\text { Components }\end{array}$} & \multicolumn{1}{c}{\begin{tabular}{c}
\multicolumn{1}{c}{ Implementation } \\
Solution
\end{tabular}} \\
\hline EHR Broker Interface & $\begin{array}{l}\text { RESTful Web services interfaces } \\
\text { complied with the APP }\end{array}$ \\
\hline Pub/Sub Server & Java RESTlet Framework \\
\hline Sync Server & $\begin{array}{l}\text { Apache Abdera Library augmented } \\
\text { with the Microsoft FeedSync } \\
\text { protocol }\end{array}$ \\
\hline Matching Engine & Apache Lucene \\
\hline EHR DB & MySQL Database 5.0 \\
\hline EHR Central API & Apache Java HTTP Client \\
\hline
\end{tabular}

\section{CONCLUSION}

The great variety and heterogeneity of existing EHR systems which apply different ways to store, access, manage and share patient information creates a problem threatening people's lives. In this paper we have proposed a solution to this problem using the cutting-edge technologies, namely, we have illustrated creation of interoperable healthcare systems using Ontology and Web Service technologies. Ontology technology enables translating between different EHR formats while Web Services technology provides an infrastructure that links all medical and health institutions and empowers them to share patient information efficiently.

The associated security and privacy issues must be addressed within the system. Addressing the security and privacy within the proposed systems is outside the scope of this paper but will be discussed in our forthcoming papers.

\section{REFERENCES}

[1] A. Cornwall, "Electronic Health Records: An International Perspective", Health Issues, no. 73, pp. 19-23, 2002.

[2] D. Rowlands, "HealthConnect: A Health Information Network for All Australians", Ed. J. E. Demetriades, G. A. Christopherson, R.M. Kolodner, Person-Centered Health Records Toward HealthePeople, pp. 242-258, 2005.

[3] http://www.abc.net.au/7.30/content/2007/s2050008.htm

[4] Accenture's Health \& Life Sciences Group, 2005, "Electronic Health Records Survey: Achieving High Performance in Health Care", retrieved

from http://www.worldcongress.com/events/nw600/pdf/Electronic\%20Healt h\%20Records\%20Survey\%20FINAL\%200705.pdf

[5] R. Hillestad, J. Bigelow, A. Bower, F. Girosi, R. Meili, R. Scoville R. Taylor, "Can electronic medical record systems transform health care? Potential health benefits, savings and costs", Health Affairs, vol. 24, no. 5, pp. 1103-1117, 2005.

[6] A.T.S. Chan, J. Cao, H. Chan, G. Young, “A Web-Enabled Framework for Smart Card Application in Health Services", Communications of the $A C M$, vol. 44, no. 9, pp. 77-82, 2001.

[7] CASPIAN, "FDA Letter Raises Questions about VeriChip Safety, Data Security", retrieved from http://www.spychips.com/devices/verichipfda-report.html, 2004.

[8] K. Albrecht, "Microchip-Induced Tumors in Laboratory Rodents and Dogs: A Review of the Literature 1990-2006", retrieved from http://www.antichips.com/cancer/albrecht-microchip-cancer-fullpaper.pdf, 2007.

[9] D. Gans, J. Kralewski, T. Hammons, B. Dowd, "Medical groups' adoption of electronic health records and information systems", Health Affairs, vol. 24, no. 5, pp. 1323-1333, 2005.

[10] T. Gruber, "Towards principles for the design of ontologies used for knowledge sharing", International Journal of Human and Computer Studies, vol. 43, no. 5-6, pp. 907-928, 1995.

[11] M. Hadzic, T. Dillon, E. Chang, "Use of Ontology Technology for Standardization of Medical Records and Dealing with Associated Privacy Issues", Proceedings of the IEEE International Conference on Industrial Technology (ICIT 2006), special session on Security and Privacy in Healthcare Informatics, India, pp. 2839-2845, 2006.

[12] R. T. Fielding, J. Gettys, J. Mogual, H. Frystyk, L. Masinter, P. Leach, T. Berners-Lee, "Hypertext Transfer Protocol -- HTTP/1.1". Retrieved from http://www.w3.org/Protocols/rfc2616/rfc2616.html, 1999.

[13] M. Nottingham, R. Sayre, "The Atom Syndication Format". Retrieved from http://tools.ietf.org/html/rfc4287, 2005

[14] C. Wu and E. Chang, "Aligning with the Web: An Atom-based Architecture for Web Services Discovery", Service-Oriented Computing and Applications, vol. 1, pp. 97 - 116, 2007

[15] J. Gregorio, B. de Hora, "The Atom Publishing Protocol". Retrieved from http://www.rfc-editor.org/rfc/rfc5023.txt, 2007. 